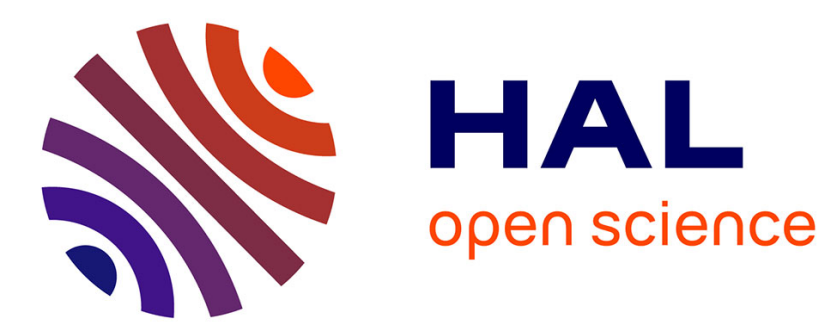

\title{
Eigenvalue variations and semiclassical concentration
}

\author{
Luc Hillairet
}

\section{To cite this version:}

Luc Hillairet. Eigenvalue variations and semiclassical concentration. Spectrum and dynamics, Apr 2008, Montréal, Canada. pp.75-87. hal-00438761

\section{HAL Id: hal-00438761 https://hal.science/hal-00438761}

Submitted on 4 Dec 2009

HAL is a multi-disciplinary open access archive for the deposit and dissemination of scientific research documents, whether they are published or not. The documents may come from teaching and research institutions in France or abroad, or from public or private research centers.
L'archive ouverte pluridisciplinaire HAL, est destinée au dépôt et à la diffusion de documents scientifiques de niveau recherche, publiés ou non, émanant des établissements d'enseignement et de recherche français ou étrangers, des laboratoires publics ou privés. 


\title{
EIGENVALUE VARIATIONS AND SEMICLASSICAL CONCENTRATION
}

\author{
LUC HILLAIRET
}

\section{INTRODUCTION}

In this paper, we aim at expliciting some relations between the behaviour of analytic eigenvalue branches of a spectral problem and concentration properties of its eigenfunctions. In order to illustrate this we consider a semiclassical Schrödinger operator $-h^{2} \Delta+V$ on $\mathbb{R}^{d}$ (in which $\Delta$ is the non-positive Euclidean Laplace operator). Viewing $h$ as an analytic parameter, this problem enters the usual perturbation theory and the eigenvalues are organised into analytic eigenbranches $E_{j}(h)$. It is then natural to address the behaviour of these eigenbranches when $h$ goes to 0 . This point of view is fundamentally different from the usual semiclassical perspective. Indeed, any analytic eigenbranch is expected to cross a typical energy window $\left[E_{1}, E_{2}\right]$ so that any semiclassical expansion in this window corresponds actually to different eigenbranches. Our point is that knowing how the eigenfunctions concentrate in the semiclassical regime yields some result on the behaviour of eigenbranches. For instance, we have the following theorem (see section 3).

Theorem 1.1. Let $E_{j}(h)$ be an analytic eigenbranch of the family of operators $-h^{2} \Delta+V$ on $L^{2}\left(\mathbb{R}^{d}\right)$. The following then holds.

(1) The eigenbranch $E_{j}(h)$ converges to some limit $E_{0}$ when $h$ tends to 0.

(2) The limit $E_{0}$ is necessarily a critical value of the potential $V$.

We will also use the same kind of ideas to prove an integrated remainder estimate for an analytic variation of metrics on a smooth compact manifold $X$. (see Thm. 4.3 in section (1).

As we have already pointed out, the study of analytic eigenbranches and semiclassical analysis are two different worlds so that such a relation between them is a priori quite surprising. However, as it will become clear later, the formula that expresses the derivative of any analytic eigenbranch is actually a semiclassical quantity. This is the fact we will be exploiting here. This idea is also used in the two recent papers $[8]$ and $[5]$. In $[8$ we deal with the limiting behaviour of analytic eigenbranches in a singular setting. The main ingredient of the proof consists in controlling certain quadratic forms evaluated on the eigenfunctions of the spectral problem under consideration. We claim here that such a control may actually be interpreted as a condition on the associated semiclassical measures. Such an interpretation is not necessarily helpful since rather little is known in general about semiclassical measures and the overall strategy of 8 relies more on direct estimates for solutions of one dimensional ODE's. In [5], a part of the proof relies on the fact that an assumption on semiclassical measures allows to prove an integrated remainder estimate of the form we will consider here. Section 1 can actually be seen as a 
formalization of this argument of [5].

Acknowledgments : The ideas in this paper grew out from several discussions with Chris Judge and Andrew Hassell, I would like to thank both of them for their stimulating influence. I would also like to thank the CRM in Montréal and the organizers of the workshop "Spectrum and Dynamics" in April, 2008 and the MSRI in Berkeley where part of this work was done. Finally, I would like to thank Gilles Carron, Yves Colin de Verdière, Patrick Gérard and Andrew Hassell who made several comments on the first version of this paper that helped me improve the results and the exposition.

\section{2. quantization And Semiclassical measures}

In this section we will briefly recall some known facts about quantization and semiclassical measures. We will use both the classical and the semiclassical theory of pseudodifferential operators (See [10, th for background on these notions)

In the case of Schrödinger operators in $\mathbb{R}^{d}$, the principal symbol of a $(h$-)pseudodifferential operator is a function in $T^{*}\left(\mathbb{R}^{d}\right)$. When working on a smooth compact Riemannian manifold, we will denote by $S^{*} X$ the quotient of $T^{*} X \backslash\{0\}$ by $(x, \xi) \sim(x, \lambda \xi), \lambda>0$. The subsets of $S^{*} X$ are the conical subsets of $T^{*} X \backslash\{0\}$. The symbol of a pseudodifferential operator $A$ is defined by testing $A$ again fast oscillatory functions. It is then a function on $T^{*} M \backslash\{0\}$. The symbol of a classical pseudodifferential operator is conically invariant and thus defines a function on $S^{*} X$.

Remark 2.1. The principal symbol of a pseudodifferential operator may also be read off from the Schwartz kernel of $A$. Observe, however, that when performing this identification, one has to pay careful attention to the density that is used (especially when dealing with varying Riemannian metrics).

A crucial point is the ability of quantizing any reasonable function on $S^{*} X$. This is done via a so-called quantization procedure. There are several possible choices, but it is useful to choose a quantization that respects positivity (see [3, ]) ]. Thus, for any function $a$ on $S^{*} X$ there exists a pseudodifferential operator $\mathrm{Op}^{+}(a)$ of order 0 whose principal symbol is $a$. If $A$ is any other pseudodifferential operator with principal symbol $a$ then $\mathrm{Op}^{+}(a)-A$ is a pseudodifferential operator of order -1 . Moreover if, $a$ is non-negative, then $\mathrm{Op}^{+}(a)$ is also non-negative, as an operator.

In the semiclassical setting, the homogeneity condition on $a$ is dropped and we may quantize any smooth function on $T^{*}(X)$ with compact support.

Using this quantization, we define the so-called semiclassical measures. We present the construction in the semiclassical setting since in the classical one we will use a non-concentration estimate rather than semiclassical measures.

We start with a Schrödinger operator on $\mathbb{R}^{d}$, and we consider a sequence of normalized eigenfunctions $u_{h}$ of the equation

$$
-h^{2} \Delta u_{h}+V(x) u_{h}=E_{h} u_{h},
$$

such that the corresponding eigenvalues $E_{h}$ converge to some non-critical energy $E_{0}$. To any compactly supported function $a$ the map $a \mapsto\left\langle\mathrm{Op}^{+}(a) u_{h}, u_{h}\right\rangle$ defines

\footnotetext{
${ }^{1}$ It is actually critical that such a quantization exists
} 
a measure that converges weakly (up to extracting a subsequence). Any such limit is called a semiclassical measure.

Remark 2.2. One could in fact associate semiclassical measures to any bounded sequence in $L^{2}$. In the sequel we will consider only semiclassical measures that are associated with eigenfunctions of the Laplace operator.

Among the basic facts satisfied by any semiclassical measure, we mention only the two following properties (see [3, 7])

(1) Any semiclassical measure is a probability measure supported on the energy surface $\Sigma_{E_{0}}:=\left\{|\xi|^{2}+V(x)=E_{0}\right\}$.

(2) Any semiclassical measure is invariant under the Hamiltonian flow associated with $|\xi|^{2}+V(x)$ on $\Sigma_{E_{0}}$.

Remark 2.3. Under suitable hypotheses, we may extend the definition to smooth functions that are not necessarily compactly supported in $T^{*} \mathbb{R}^{d}$. In particular, for a sequence of eigenfunctions with energy $E_{h}$ converging to $E_{0}$, the quantity $\int\left\|\nabla u_{h}\right\|^{2} d x$ is uniformly bounded. This allows to prove that $\int|\xi|^{2} d \mu<+\infty$ and then that

$$
\int|\xi|^{2} d \mu=\lim _{h \rightarrow 0} \int\left\|\nabla u_{h}\right\|^{2} d x
$$

In the classical setting we can also define semiclassical measures. The main difference is that instead of a small parameter going to zero, we have to consider a sequence of eigenfunctions with eigenvalues growing to infinity and we recover a probability measure on $S^{*} X$. It can be pointed out that the estimate in corollary 4.7 that we use in section 1 is a kind of non-concentration estimate. Indeed, such an estimate would give some information on any semiclassical measure when passing to the high energy limit. The assumptions on semiclassical measure in section 3 and on geometric control in section 1 are thus very similar in nature.

\section{SCHRÖDINGER OPERATORS FROM THE ANALYTIC VIEWPOINT}

In this section we consider the semiclassical Schrödinger equation in $X=\mathbb{R}^{d}$. The eigenvalue problem consists in looking for eigenfunctions $u_{h}$ and eigenvalues $E_{h}$ such that

$$
-h^{2} \Delta u_{h}+V(x) u_{h}=E_{h} u_{h},
$$

in which $\Delta$ is the (non-positive) Laplace operator associated with the Euclidean metric. Let us assume that $V$ is smooth and confining (i.e. $\lim _{|x| \rightarrow \infty} V(x)=+\infty$ ) so that there is a complete orthonormal set of eigenfunctions and the corresponding eigenvalues grow to infinity.

This eigenvalue problem may be put into a generalized eigenvalue problem in the sense of Kato (cf [1). We change notations by letting $h=t$ thus emphasizing that we will now consider an analytic perturbation problem. An integration by parts leads to the following equivalent problem

$$
q_{t}\left(u_{t}, v\right)=E_{t} \cdot n(u, v),
$$

In which $q_{t}$ is the quadratic form

$$
q_{t}(u)=t^{2} \int|\nabla u(x)|^{2} d x+\int V(x)|u(x)|^{2} d x
$$


defined on $\mathcal{D}=H^{1}(X) \cap L^{2}\left(\left(1+V(x)^{2}\right)^{\frac{1}{2}} d x\right)$ and $n$ is the standard Riemannian quadratic form on $L^{2}(X)$. Observe that although $q_{t}$ is real-analytic for $t \in \mathbb{R}, \mathcal{D}$ is the form domain of $q_{t}$ only for $t \neq 0$. Consequently, usual analytic perturbation theory tells us that the eigenvalues organize into real-analytic eigenbranches for $t \in(0,1]$. A standard question in this setting is to address the limiting behaviour of these eigenbranches when $t$ goes down to 0 .

Theorem 3.1. Let $E_{t}$ be an eigenvalue branch of (2), then $E_{t}$ converges to a limit $E_{0}$ when $t$ goes to 0 . Moreover, this limit $E_{0}$ is a critical value of $V$.

Proof. Let $E_{t}$ be an eigenvalue branch and $u_{t}$ the corresponding normalized eigenvector branch. The derivative of $E_{t}$ may be computed by differentiating eq. (2) (see [9]), this yields

$$
\dot{E}_{t}=2 t \int_{X}\left|\nabla u_{t}\right|^{2}(x) d x .
$$

This expression is obviously non-negative so that $E_{t}$ is a non-decreasing function of $t$. Since, for any $t$ we have $E_{t} \geq \min _{X} V(x), E_{t}$ has a limit $E_{0}$ when $t$ goes to 0 .

The condition on concentration of semiclassical measures then enters in the following lemma.

Lemma 3.2. Assume that for any semiclassical measure $\mu$ at the energy $E_{0}$,

$$
\text { (C) } \int_{\Sigma_{E_{0}}}|\xi|^{2} d \mu(x)>0 .
$$

Then, for any eigenbranch $\left(E_{t}, u_{t}\right)$ such that $\lim E_{t}=E_{0}$,

$$
\exists c>0, \liminf _{t \rightarrow 0} t^{2} \cdot \int_{X}\left|\nabla u_{t}(x)\right|^{2} d x \geq c .
$$

Assuming this lemma, we finish the proof of the theorem. If we suppose, for a contradiction, that $E_{0}$ satisfies condition $(C)$, then using (3), and (4) we get

$$
\dot{E}_{t} \geq \frac{2 c}{t}
$$

and this is a contradiction since $E_{t}$ has to converge when $t$ goes down to 0 . So condition $(C)$ is not fulfilled and there exists a semiclassical measure at energy $E_{0}$ such that

$$
\int_{\Sigma_{E_{0}}}\left|\xi^{2}\right| d \mu=0 .
$$

This implies that the support of $\mu$ is contained in the level set $\{\xi=0\} \subset \Sigma_{E_{0}}$. Since $d \mu$ has to be invariant by the hamiltonian flow

$$
\left\{\begin{array}{rrr}
\dot{x} & = & 2 \xi \\
\dot{\xi} & = & -\nabla V(x)
\end{array}\right.
$$

this forces $E_{0}$ to be a critical value of $V$.

It remains to prove lemma 3.2 .

Proof of Lemma 3.8. As it is standard in this kind of settings (see [6] or [2]), we actually work in the reverse direction, assuming that (4) is not true. We may thus 
find a sequence $t_{n}$ going to 0 and corresponding eigenfunctions and eigenvalues $E_{t_{n}}$ and $u_{t_{n}}$, such that

$$
t_{n}^{2} \int_{X}\left|\nabla u_{t_{n}}(x)\right|^{2} d x \leq \frac{1}{n}
$$

We now change notations again and let $t_{n}=h$ since the rest of the argument is of semiclassical nature (and, as usual in this setting $h$ actually stands for $h_{n}$ ). We thus have

$$
0 \leq h^{2} \int_{X}\left|\nabla u_{h}(x)\right|^{2} d x \leq \varepsilon(h) .
$$

where $\varepsilon(h)$ is some function going to 0 with $h$. The sequence $u_{h}$ is $L^{2}$ normalized so that we may extract subsequences and find associated semiclassical measures at energy $E_{0}$ since $\lim E_{h}=E_{0}$. Let $d \mu$ be one of these semiclassical measures, by definition (see remark 2.3 above)

$$
\lim _{h \rightarrow 0} h^{2} \cdot \int_{X}\left|\nabla u_{h}(x)\right|^{2} d x=\int_{\Sigma_{E_{0}}}\left|\xi^{2}\right| d \mu,
$$

where the limit is understood along the subsequence defining $\mu$. Putting (5) and (6) together yields

$$
\int_{\Sigma_{E_{0}}}\left|\xi^{2}\right| d \mu=0
$$

thus finishing the proof of the lemma.

Remark 3.3. Since the energy surface is defined by $|\xi|^{2}+V(x)=E_{0}$, we can replace everywhere

$$
\int_{\Sigma_{E_{0}}}\left|\xi^{2}\right| d \mu
$$

by

$$
\int_{\Sigma_{E_{0}}}\left(E_{0}-V(x)\right) d \mu
$$

In dimension 1 this result can be refined using that the spectrum of a onedimensional Schrödinger operator is known to be simple. If we assume that the potential $V$ has only non-degenerate minima, then the spectrum near the bottom of the energy is known (see [i ) and there is an infinite number of eigenvalues close to the minimum. Since the eigenvalue branches cannot cross, the non-degenerate minimum is then only possible limit?

\section{INTEGRATED REMAINDER ESTIMATES}

In this section, we consider a smooth compact manifold $X$ of dimension $d \geq 2$ and a real-analytic family of Riemannian metrics $\left(g_{\tau}\right)_{\tau \in[-1,1]}$. We denote by $\Delta_{\tau}$ the Laplace operator associated with $g_{\tau}$ and by $E_{j}(\tau)$ the associated analytic branches of eigenvalues. We will also denote by $\langle\cdot, \cdot\rangle_{\tau}$ the scalar product on $L^{2}\left(X, g_{\tau}\right)$. We now define the usual following counting functions :

\footnotetext{
${ }^{2}$ I would like to thank San Vũ Ngọc and Frédéric Faure for pointing out that, in the onedimensional case, the simplicity of the spectrum allows to improve the result.
} 


$$
\begin{aligned}
N(\tau, E) & =\sharp\left\{j \mid E_{j}(\tau) \leq E\right\}, \\
R_{M}(\tau, E) & =\sharp\left\{j \mid E_{j}(\tau) \in(E-M, E+M]\right\} \\
& =N(\tau, E+M)-N(\tau, E-M) .
\end{aligned}
$$

Remark 4.1. Observe that since $\left(g_{\tau}\right)$ is analytic in $\tau$, the scalar products $\langle\cdot, \cdot\rangle_{\tau}$ define equivalent norms. The same is true at the level of $H^{1}$ : the norms $\|\cdot\|_{H^{1}\left(X, g_{\tau}\right)}$ defined by :

$$
\|u\|_{H^{1}\left(X, g_{\tau}\right)}^{2}=\int_{M} g_{\tau}\left(\nabla_{\tau} u, \nabla_{\tau} u\right) d \operatorname{vol}_{g_{\tau}}+\|u\|_{\tau}^{2},
$$

are all equivalent.

The following lemma expresses the variation of $E_{t}$ using a family of pseudodifferential operators. It also serves as a definition of the family $A_{\tau}$ that will be used in the rest of the paper.

Lemma 4.2. There exists a real-analytic family of symbols $a_{\tau}$, and, for any $\tau_{0}$, there exists a constant $C$ such that, for any normalized eigenbranch $\left(E_{n}(\tau), u_{n}(\tau)\right)$ we have

$$
\forall|\tau| \leq \tau_{0},\left|\frac{\dot{E}_{n}(\tau)}{E_{n}(\tau)}-\left\langle O p^{+}\left(a_{\tau}\right) u_{n}(\tau), u_{n}(\tau)\right\rangle_{\tau}\right| \leq C E_{n}(\tau)^{-\frac{1}{2}} .
$$

We will denote by $A_{\tau}=\mathrm{Op}^{+}\left(a_{\tau}\right)$.

Proof. First, by differentiating the eigenvalue equation

$$
E_{n}(\tau)=\left\langle\Delta_{\tau} u_{n}(\tau), u_{n}(\tau)\right\rangle_{\tau},
$$

we find a second-order differential operator $\dot{\Delta}_{\tau}$ such that

$$
\dot{E}_{n}(\tau)=\left\langle\dot{\Delta}_{\tau} u_{n}(\tau), u_{n}(\tau)\right\rangle_{\tau} .
$$

(Observe that since the branch is normalized and using the eigenvalue equation we have that $\langle\Delta \dot{u}, u\rangle=E\langle\dot{u}, u\rangle=0)$. Denote by $a_{\tau}$ the principal symbol of $\dot{\Delta}_{\tau}\left(\Delta_{\tau}+1\right)^{-1}$, and let $A_{\tau}=\mathrm{Op}^{+}\left(a_{\tau}\right)$. By definition $R_{\tau}=\dot{\Delta}_{\tau}\left(\Delta_{\tau}+1\right)^{-1}-A_{\tau}$ is an analytic family of pseudodifferential operators of order -1 . In particular, $R_{\tau}$ is uniformly bounded from $L^{2}$ into $H^{1}$. This yields the bound

$$
\left|\frac{\dot{E}_{n}(\tau)}{E_{n}(\tau)+1}-\left\langle\mathrm{Op}^{+}\left(a_{\tau}\right) u_{n}(\tau), u_{n}(\tau)\right\rangle_{\tau}\right| \leq C E_{n}(\tau)^{-\frac{1}{2}} .
$$

We thus get

$$
\left|\frac{\dot{E}_{n}(\tau)}{E_{n}(\tau)}-\left\langle\left(1+E_{n}^{-1}\right) \mathrm{Op}^{+}\left(a_{\tau}\right) u_{n}(\tau), u_{n}(\tau)\right\rangle_{\tau}\right| \leq C E_{n}(\tau)^{-\frac{1}{2}}\left(1+E_{n}^{-1}\right),
$$

from which the claimed bound follows since $\left|\left\langle\mathrm{Op}^{+}\left(a_{\tau}\right) u_{n}(\tau), u_{n}(\tau)\right\rangle_{\tau}\right|$ is uniformly bounded.

The main result of this section is then the following theorem (the notion of geometric control is defined in definition 4.4 in the following section). 
Theorem 4.3. Suppose that $a_{0}$ is non-negative and that there exists $\varepsilon>0$ such that the subset $\left\{(x, \xi) \in S^{*} X, a_{0}(x, \xi)>\varepsilon\right\}$ geometrically controls $\left(S^{*} X, g_{0}\right)$, then for any fixed $M$, there exists $\tau_{0}, E_{0}$ and $K$ such that

$$
\forall E>E_{0}, \int_{-\tau_{0}}^{\tau_{0}} R_{M}(t, E) d t \leq K E^{\frac{d}{2}-1} .
$$

The universal remainder in Weyl's law gives that for a fixed $t, R_{M}(t, E)=$ $O\left(E^{\frac{d-1}{2}}\right)$ as $E$ goes to infinity. The theorem thus says that averaging with respect to the perturbation greatly improves this estimate.

The rest of the section is devoted to the proof of this theorem. The central part of the argument is actually interesting by itself and consists in proving a somewhat explicit uniform control estimate.

4.1. A uniform control estimate. We begin by recalling the notion of geometric control observing that since the velocity is constant along a geodesic, the geodesic flow of a Riemannian metric is well-defined on $S^{*} X$.

Definition 4.4. We say that an open subset $\mathcal{U}$ of $S^{*} X$ geometrically controls $\left(S^{*} X, g\right)$ if for any $(x, \xi) \in S^{*} X$, there exists $T \in \mathbb{R}$ and $\left(x_{0}, \xi_{0}\right) \in \mathcal{U}$ such that $(x, \xi)=\Phi^{T}\left(x_{0}, \xi_{0}\right)$ where $\Phi \cdot(.,$.$) denotes the geodesic flow of g$.

It is well-known that geometric control implies a non-concentration estimate for eigenfunctions. More precisely, if $U$ is an open subset of $X$ such that $\mathcal{U}=S^{*} U$ geometrically controls $\left(S^{*} X, g\right)$ then, there exists some positive $c$ and an energy $E_{0}$ such that

$$
\int_{U}\left|u_{n}\right|^{2} d v o l_{g} \geq c \int_{X}\left|u_{n}\right|^{2} d v o l_{g},
$$

for any eigenfunction $u_{n}$ of the Laplace operator associated with $g$ such that $E_{n} \geq$ $E_{0}$. (Actually the restriction on $E$ for this estimate may be released using the principle of unique continuation for solutions of second order elliptic PDE's see remark 4.6 below)

Such a control estimate can be proved by a contradiction argument relying on known properties of semiclassical measures ([6], [2] for the scheme of such an argument). Such an approach can probably be adapted to get a control estimate that is uniform in $\tau$. We propose here a slightly different proof that also allows us to get some uniform control on $c$.

Proposition 4.5. Let $\mathcal{U}$ be an open subset of $S^{*} X$ that geometrically controls $\left(S^{*} X, g_{0}\right)$. There exists $\tau_{0} \in \mathbb{R}, E_{0} \in \mathbb{R}$, a zeroth-order pseudodifferential operator $\Pi$ on $X$ and $c>0$ such that :

(1) The symbol $\pi$ of $\Pi$ is supported in $\mathcal{U}$ and, $0 \leq \pi(x, \xi) \leq 1$ on $S^{*} X$.

(2) For any $|\tau| \leq \tau_{0}$ and any $u$ eigenfunction of $\Delta_{\tau}$ with energy greater than $E_{0}$ then the following non-concentration estimate holds :

$$
\|\Pi u\|_{\tau} \geq c\|u\|_{\tau} .
$$

Remark 4.6. Estimate (8) may be extended to any eigenfunction, without any restriction on the energy. Such an extension requires that one knows that an eigenfunction of a Laplace operator cannot vanish on an open subset (thus proving the control estimate for any finite number of eigenfunctions). The principle of unique continuation gives this property for eigenfunctions of the Laplace operator. It is not 
clear to the author if this non-vanishing property holds in the cotangent bundle. It can also be observed that, strictly speaking, the formulation with the microlocal cutoff only has a meaning in the high energy limit since the microlocal cutoff is defined modulo smoothing operators.

Proof. Let $(x, \xi) \in S^{*} X$, the geometric control assumption implies that there exists some $T(=T(x, \xi))$ and $\left(x_{0}, \xi_{0}\right) \in \mathcal{U}$ such that $\Phi_{0}^{T}\left(x_{0}, \xi_{0}\right)=(x, \xi)$. Using the continuity properties of solutions of an O.D.E. both with respect to initial conditions and with respect to parameters, we can find

- some $\tau_{\max }(x, \xi)$,

- two conical neighbourhoods of $(x, \xi): \mathcal{V}=\mathcal{V}_{(x, \xi)}$ and $\mathcal{W}=\mathcal{W}_{(x, \xi)}$,

- a conical neighbourhood $\tilde{\mathcal{U}}=\tilde{\mathcal{U}}_{(x, \xi)}$ of $\left(x_{0}, \xi_{0}\right)$

such that $\mathcal{V}$ is compactly included in $\mathcal{W}$ and $\Phi_{\tau}^{-T}(\mathcal{W}) \subset \tilde{\mathcal{U}} \Subset \mathcal{U}$ for any $|\tau| \leq$ $\tau_{\max }(x, \xi)$. Since $S^{*} X$ is compact, we can find a finite collection $\left(\left(x_{i}, \xi_{i}\right)\right)_{1 \leq i \leq N}$ such that $S^{*} X=\bigcup_{i=1}^{N} \mathcal{V}_{\left(x_{i}, \xi_{i}\right)}$. We now use the index $i$ to denote any quantity formerly indexed by (or attached to) $\left(x_{i}, \xi_{i}\right)$. Denote by $\tau_{0}$ the minimum of all $\tau_{\max }\left(x_{i}, \xi_{i}\right)$. We can then find a smooth partition of unity $\pi_{i}$ such that each $\pi_{i}$ is identically 1 in $\mathcal{V}_{i}$ and identically 0 outside $\mathcal{W}_{i}$. Note that, by construction,

$$
\forall|\tau| \leq \tau_{0}, \bigcup_{i=1}^{N} \Phi_{\tau}^{-T_{i}}\left(\mathcal{W}_{i}\right) \subset \bigcup_{i=1}^{N} \tilde{\mathcal{U}}_{i} \Subset \mathcal{U} .
$$

We denote by $\pi_{0}$ a smooth function which is identically 1 in $\bigcup_{i=1}^{N} \tilde{\mathcal{U}}_{i}$ and 0 outside $\mathcal{U}$. Denote by $\Pi_{i}=\mathrm{Op}^{+}\left(\pi_{i}\right)$, any by $U_{\tau}(T)=\exp i T \sqrt{\Delta_{\tau}}$, we claim that for any $i>0$, and for any $|\tau| \leq \tau_{0}$, the operator $R_{i}=\Pi_{i} U_{\tau}\left(T_{i}\right)\left(\mathrm{Id}-\Pi_{0}\right)$ is smoothing. Indeed, its wave-front set is included

$$
\left\{(y, \eta, x, \xi) \mid(y, \eta) \in \operatorname{supp} .\left(\pi_{i}\right),(x, \xi) \in \operatorname{supp} .\left(1-\pi_{0}\right),(y, \eta)=\Phi_{\tau}^{T_{i}}(x, \xi)\right\},
$$

which is empty by construction. In particular, each $R_{i, \tau}$ is bounded from $H^{-1}(X)$ into $L^{2}(X)$. Moreover, a careful analysis of the construction of the Hadamard parametrix (as it is for instance presented in [1]) shows that there exists a uniform constant $C$ such that :

$$
\forall|\tau| \leq \tau_{0}, \forall w \in H^{-1}(X),\left\|R_{i} w\right\|_{L^{2}\left(X, g_{0}\right)} \leq C\|u\|_{H^{-1}\left(X, g_{0}\right)},
$$

(see the appendix for a brief sketch of proof).

Since the $\left(\pi_{i}\right)_{i>1}$ are a partition of unity, $\sum_{i>1} \Pi_{i}=$ Id. We thus have

$$
\|u\|_{0}^{2}=\sum_{i=1}^{N}\left\langle\Pi_{i} u, u\right\rangle_{0} .
$$

Using that $u$ is an eigenfunction of $\Delta_{\tau}$, we have

$$
\begin{aligned}
\left|\left\langle\Pi_{i} u, u\right\rangle_{0}\right| & =\left|\left\langle\Pi_{i} U_{\tau}\left(T_{i}\right) u, u\right\rangle_{0}\right| \\
& \leq\left|\left\langle\Pi_{i} U_{\tau}\left(T_{i}\right) \Pi_{0} u, u\right\rangle_{0}\right|+\left|\left\langle R_{i} u, u\right\rangle_{0}\right| .
\end{aligned}
$$

We use Cauchy-Schwarz inequality on both terms and the following facts on the norm of the different operators :

- $\Pi_{i}$ is bounded from $L^{2}(X)$ to itself,

- $U_{\tau}(T)$ is an isometry from $L^{2}(X)$,

- and (10). 
This yields :

$$
\left|\left\langle\Pi_{i} u, u\right\rangle_{0}\right| \leq\left(c_{i}\left\|\Pi_{0} u\right\|_{0}+C\|u\|_{H^{-1}\left(X, g_{0}\right)}\right) \cdot\|u\|_{0} .
$$

Using that the $H^{-1}\left(X, g_{\tau}\right)$ norms are all equivalent and that $\|u\|_{H^{-1}\left(X, g_{\tau}\right)}^{2}=$ $(1+E)^{-1}\|u\|_{\tau}^{2}$ when $u$ is an eigenfunction of $\Delta_{\tau}$, we get

$$
\left|\left\langle\Pi_{i} u, u\right\rangle_{0}\right| \leq\left(c_{i}\left\|\Pi_{0} u\right\|+C(1+E)^{-\frac{1}{2}}\|u\|_{0}\right) \cdot\|u\|_{0}
$$

for a constant $C$ independent of $\tau \in\left[-\tau_{0}, \tau_{0}\right]$.

Summing all these inequalities and denoting by $\tilde{c}$ the maximum of the $c_{i}$ we obtain:

$$
\|u\|_{0} \leq(N \tilde{c})\left\|\Pi_{0} u\right\|+C N(1+E)^{-\frac{1}{2}}\|u\|_{0}
$$

This yields the estimate in the proposition with $\Pi=\Pi_{0}$ and $c^{-1}=2 \tilde{c} N$ as soon as $C N(1+E)^{-\frac{1}{2}}$ is less than $\frac{1}{2}$.

This proposition has the following useful corollary.

Corollary 4.7. Let $a_{\tau}$ be an analytically dependent family of non-negative symbols of order 0 such that $\left\{a_{0}(x, \xi)>\varepsilon\right\}$ geometrically controls $\left(S^{*} X, g_{0}\right)$ then there exist positive $\kappa, \tau_{0}$ and $E_{0}$ such that, for any $|\tau| \leq \tau_{0}$ and any $u$ eigenfunction of $\Delta_{\tau}$ with energy greater than $E_{0}$ then

$$
\left\langle A_{\tau} u, u\right\rangle_{0} \geq \kappa\|u\|_{\tau}^{2},
$$

where $A_{\tau}$ denotes $\mathrm{Op}^{+}\left(a_{\tau}\right)$.

Remark 4.8. In the following proof, we will use several times the fact that, for any symbols of order $0, \mathrm{Op}^{+}(b c)-\mathrm{Op}^{+}(b) \circ \mathrm{Op}^{+}(c)$ is a pseudodifferential operator of order -1 . We will denote by $R$ any operator arising from this kind of operation. Observe that if $b$ and $c$ depend analytically on $\tau$, then $R$ also depends analytically on $\tau$.

Proof. Let $\tau_{0}, \tilde{E}_{0}$ and $\Pi$ be given by applying Proposition 4.5 to $\mathcal{U}=\left\{a_{0}(x, \xi)>\varepsilon\right\}$. If needed, we restrict $\tau_{0}$ so that, for any $|\tau| \leq \tau_{0}, a_{\tau}$ is bounded below by $\frac{\varepsilon}{2}$ on $\mathcal{U}$. Observe that

$$
\begin{aligned}
\left\langle A_{\tau} u, u\right\rangle_{0} & =\left\langle A_{\tau} \Pi^{2} u, u\right\rangle_{0}+\left\langle A_{\tau}\left(\mathrm{Id}-\Pi^{2}\right) u, u\right\rangle_{0} \\
& =\left\langle\mathrm{Op}^{+}\left(a_{\tau} \pi^{2}\right) u, u\right\rangle_{0}+\left\langle\mathrm{Op}^{+}\left(a_{\tau}\left(1-\pi^{2}\right) u, u\right\rangle_{0}+\left\langle R_{\tau} u, u\right\rangle_{0}\right. \\
& \geq\left\langle\mathrm{Op}^{+}\left(a_{\tau} \pi^{2}\right) u, u\right\rangle_{0}+\left\langle R_{\tau} u, u\right\rangle_{0},
\end{aligned}
$$

where the last inequality holds because $\pi(x, \xi) \leq 1$ and we have chosen a positive quantization. Since the support of $\pi$ is included in $\mathcal{U}$ we have

$$
\forall(x, \xi) \in S^{*} X, a_{\tau}(x, \xi) \pi^{2}(x, \xi) \geq \frac{\varepsilon}{2} \pi^{2}(x, \xi),
$$

which is quantized into

$$
\begin{aligned}
\left\langle\mathrm{Op}^{+}\left(a_{\tau} \pi^{2}\right) u, u\right\rangle_{0} & \geq \frac{\varepsilon}{2}\left\langle\mathrm{Op}^{+}\left(\pi^{2}\right) u, u\right\rangle_{0} \\
& \geq \frac{\varepsilon}{2}\|\Pi u\|_{0}^{2}+\left\langle R_{\tau} u, u\right\rangle_{0},
\end{aligned}
$$

with another remainder operator $R_{\tau}$. Putting these two inequalities together, we obtain

$$
\langle A u, u\rangle_{0} \geq \frac{\varepsilon}{2}\|\Pi u\|_{0}^{2}+\left\langle R_{\tau} u, u\right\rangle_{0}
$$


Since $R$ is of order -1 and the norms on $H^{-1}$ associated with $\left(g_{\tau}\right)_{|\tau| \leq \tau_{0}}$ are uniformly equivalent, there exists some uniform $C$ such that

$$
\left|\left\langle R_{\tau} u, u\right\rangle_{0}\right| \leq C E^{-\frac{1}{2}}\|u\|_{\tau} .
$$

Using Proposition 4.5, for $|\tau| \leq \tau_{0}$ and $E \geq E_{0}$, we have $\|\Pi u\|_{0} \geq c_{1}\|\Pi u\|_{\tau} \geq$ $c_{2}\|u\|_{\tau}$ so that we get

$$
\left\langle A_{\tau} u, u\right\rangle \geq\left(c \frac{\varepsilon}{2}-C E^{-\frac{1}{2}}\right) \cdot\|u\|_{\tau}^{2}
$$

The corollary then holds with $\kappa=\frac{c \varepsilon}{4}$ as soon as $E$ is large enough so that $C E^{-\frac{1}{2}} \leq$ $\frac{c \varepsilon}{4}$.

4.2. Proof of Theorem 4.3. We consider normalized eigenbranches and we start from the formula for the derivative of the eigenfunctions : (in the following equation, everything except the constant $C$ depends on $\tau$ )

$$
\frac{d}{d \tau} \ln (E) \geq\langle A u, u\rangle-C E^{-\frac{1}{2}}
$$

Under the assumptions of the theorem, we may use proposition 4.5 and its corollary. This provides us with $\kappa>0, \tau_{0}$ and $E_{0}$ such that

$$
\begin{gathered}
\forall|\tau| \leq \tau_{0}, \forall E(\tau) \geq E_{0}, \\
\frac{d}{d \tau} \ln (E) \geq \kappa .
\end{gathered}
$$

The rest of the argument is merely reproduced from [5].

Denote by $R_{M}(E)=\int_{-\tau_{0}}^{\tau_{0}} R_{M}(t, E) d t$. A straightforward manipulation of the integral yields :

$$
R_{M}(E)=\sum_{j} \operatorname{Leb}\left\{\tau \mid E_{j}(\tau) \in(E-M, E+M]\right\}
$$

where Leb denotes the Lebesgue measure. We consider now $E$ such that $E-M \geq$ $E_{0}$. According to (14), any eigenbranch that crosses the energy $E-M$ has to do it in an increasing manner. This implies that any eigenbranch for which the set $I_{j}:=\left\{t \mid E_{j}(\tau) \in(E-M, E+M]\right\}$ is not empty satisfies $E_{j}\left(-\tau_{0}\right) \leq E+M$ and $E_{j}\left(\tau_{0}\right) \geq E-M$. In particular, the sum in (15) has only a finite number of positive terms, and moreover, this number is bounded by $N_{-\tau_{0}}(E+M)$ which is bounded by $C E^{\frac{d}{2}}$ according to Weyl's law. Estimate (14) also implies that $I_{j}$ is an interval whose extremities we denote by $\tau_{j}^{-}$and $\tau_{j}^{+}$. Integrating (14) we obtain

$$
\begin{aligned}
\kappa\left(\tau_{j}^{+}-\tau_{j}^{-}\right) & \leq \ln \left(\frac{E_{j}\left(\tau_{j}^{+}\right)}{E_{j}\left(\tau_{j}^{-}\right)}\right) \\
& \leq \ln \left(\frac{E+M}{E-M}\right) \\
& \leq \frac{C}{E}
\end{aligned}
$$

\footnotetext{
${ }^{3}$ We do not specify whether $I_{j}$ is open or closed at each of its extremity since this fact depends on whether $\tau_{j}^{ \pm}$coincide or not with $\pm \tau_{0}$, and it is irrelevent when computing the Lebesgue measure.
} 
as soon as $E$ is large enough (indepently of $j$ ). This bound each term of the sum in (15), since the number of terms is also bounded explicitly using Weyl's law, we obtain

$$
R_{M}(E) \leq C \cdot E^{\frac{d}{2}-1} .
$$

Remark 4.9. The fact that $a_{0}$ is non-negative implies in particularly that the derivative of $\operatorname{vol}_{g_{\tau}} X$ is increasing. In particular, the variation cannot be volumepreserving.

Remark 4.10. Corollary 4.7) implies that for any semiclassical measure associated with $\Delta_{\tau}$, the following lower bound holds :

$$
\int a d \mu \geq \kappa
$$

If we replace the control assumption in Thm.4.3) by the following (weaker) assumption :

$$
\begin{gathered}
\forall \tau, \forall \mu \text { semiclassical measure associated with } \Delta_{\tau}, \\
\qquad a d \mu>0,
\end{gathered}
$$

then, following the same lines of proof, we would get a 'almost-everywhere' result in the spirit of [5].

Remark 4.11. This result can probably be extended to manifolds with boundary with the precaution that, in this case we would have to control the possible escape of the mass to the boundary.

\section{Examples :}

(1) A natural variation to look at is to simply take $g_{\tau}=\exp (\tau) g_{0}$. In this case the control assumption is trivially satisfied so that we get a uniform integrated remainder. However, this is not a new result since looking at a fixed energy interval and dilating the volume is equivalent to fixing the volume and dilating the energy interval so that the integrated remainder is actually a regularized remainder in Weyl's law. And it is known that integrating the remainder in Weyl's law with respect to the energy yields a much better estimate than the universal remainder term.

(2) For a more general conformal variation on a surface $(d=2)$ we have $g_{\tau}:=$ $(1+\tau f(x))^{2} g_{0}$, the eigenvalue problem can be put in the form

$$
\int_{X} \nabla_{0} u \nabla_{0} \phi d v o l_{g_{0}}=E \int_{X} u \phi(1+\tau f(x))^{2} d v o l_{g_{0}} .
$$

This gives $a_{0}(x, \xi)=2 f(x)$. Choosing $f$ non-negative and supported in a control region yields examples where the theorem applies.

(3) On the contrary, the variation considered in [5] on a Donnelly surface doesn't satisfy the control estimate because of the cylinder of periodics orbits and of periodic geodesic in the hyperbolic wings. 


\section{ApPendix : Uniform HAdAmard PARAmetrix}

The aim of this appendix is to give a (rather sketchy) justification of the uniform estimate 10 .

Let $X$ be a smooth manifold without boundary and $g_{\tau}$ a real-analytic family of riemannian metrics.

Denote by $U_{\tau}(s)$ the propagator of the half-wave equation associated with $\Delta_{\tau}$ :

$$
U_{\tau}(s)=\exp \left(i s \sqrt{\Delta_{\tau}}\right),
$$

and denote by $k_{\tau}(s, x, y)$ its Schwartz kernel. This kernel $k_{\tau}(s, x, y)$ is obtained by restricting the Schwartz kernel of $\cos \left(s \sqrt{\Delta_{\tau}}\right)$ to the region $\sigma>0$ of the cotangent bundle $T^{*}(\mathbb{R} \times X \times Y)$

We start from the Hadamard parametrix construction as explained for instance in [1]. Concretely, for $s$ less than some small $\varepsilon$ and $x, y$ such that $d_{\tau}(x, y) \leq 2 \varepsilon$, we make the ansatz

$$
\tilde{k}_{N}(\tau, s, x, y)=\sum_{1}^{N} u_{k}(\tau, x, y) T_{\alpha_{d}+k}\left(s^{2}-d_{\tau}^{2}(x, y)\right),
$$

in which the $u_{k}$ are smooth functions of $x, y$ and $T_{\alpha+k}(z)$ are oscillatory distributions of $\mathbb{R}$ with wave-front in $\{(z=0, \zeta>0)\}$. The coefficients $u_{k}$ satisfy some transport equations from which it is straightforward that they also depend smoothly on $\tau$. We fix a smooth cutoof function $\rho:=\rho\left(d_{\tau}(x, y)\right)$ that cuts off in $d_{\tau}(x, y) \leq 2 \varepsilon$ and we let $k_{N}=\tilde{k}_{N} \times \rho$ A computation then yields that $k_{N}$ solves the half-wave equation up to a remainder term $r_{N}(\tau, s, x, y)$ which is $\mathcal{C}^{l(N)}$ where $l(N)$ tends to infinity with $N$. Applying Duhamel's principle, we get

$$
k(\tau, s, x, y)-k_{N}(\tau, s, x, y)=\int_{0}^{s} k\left(\tau, s-s^{\prime}, x, w\right) r_{N}\left(\tau, s^{\prime}, w, y\right) d s^{\prime} d w
$$

Here again, a detailed analysis shows that, for $s \leq \varepsilon$ and for any $N$, we finally have

$$
k(\tau, s, x, y)=k_{N}(\tau, s, x, y)+R_{N}(\tau, s, x, y),
$$

in which everything is smooth in $\tau$, and $R_{N}$ is $\mathcal{C}^{l(N)}$. Since $k_{N}$ has a fixed order as a distribution of $(s, x, y)$, applying it to a $C^{l}$ function gives a $C^{l-\beta}$ with a loss $\beta$ that is independant of $N$.

We now fix some $T$, and we use the semigroup properties of $U$ to write

$$
U_{\tau}(T)=U_{\tau}(\delta) \circ U_{\tau}(\delta) \circ \cdot \circ U_{\tau}(\delta),
$$

with a fixed small $\delta$. We now plug into this expression the decomposition $U_{\tau}=K_{N}+$ $R_{N}$. Expanding everything gives a large sum in which each term is a composition of $K_{N}$ factors with $R_{N}$ factors. We address the regularity of each term. Each term has regularity $N-p \beta$ where $p$ is the number of $K_{N}$ factors. Thus any term except the one with only $K_{N}$ factors may be made as regular as wanted by choosing $N$ large enough. Moreover, everything is regular in $\tau$.

It remains to address the term with only $K_{N}$ factors. Each $K_{N}$ is explicit and this composition is adressed using stationary phase. It is somewhat tedious in the general case because for instance of conjugate points. However it is much simpler when we have an initial and final microlocal cutoff that destroys everything in the wave-front set. Indeed a uniform non-stationary phase then applies that yields a uniform $C^{m}$ bound for any $m$. 


\section{REFERENCES}

[1] P. Bérard. On the wave equation on a compact Riemannian manifold without conjugate points. Math. Z., 155(3):249-276, 1977.

[2] N. Burq and M. Zworski. Geometric control in the presence of a black box. Jour. Amer. Math. Soc., 17(2), 443-471, 2004.

[3] Y. Colin de Verdière. Ergodicité et fonctions propres du laplacien. Comm. Math. Phys., 102(3):497-502, 1985.

[4] M. Dimassi and J. Sjöstrand. Spectral asymptotics in the semi-classical limit, volume 268 of London Mathematical Society Lecture Note Series. Cambridge University Press, Cambridge, 1999.

[5] A. Hassell. Ergodic Billiards that are not Quantum Unique Ergodic, with an appendix by A. Hassell and L. Hillairet http://arxiv.org/abs/0807.0666v3, to appear in Ann. of Math.,2008.

[6] A. Hassell, L. Hillairet and J. Marzuola. Eigenfunction concentration in polygonal billiards, Comm. Part. Diff. Eq., 34(5):475-485, 2009.

[7] B. Helffer, A. Martinez, and D. Robert. Ergodicité et limite semi-classique. Comm. Math. Phys., 109(2):313-326, 1987.

[8] L. Hillairet and C. Judge. The eigenvalues of a domains with small slits. Trans. Amer. Math. Soc., to appear, 2008.

[9] T. Kato. Perturbation theory for linear operators. Classics in Mathematics. SpringerVerlag, Berlin, 1995. Reprint of the 1980 edition.

[10] M. Taylor. Pseudodifferential Operators, volume 34 of Princeton Mathematical Series. Princeton University Press, Princeton, N.J., 1981.

Laboratoire de Mathématiques Jean Leray, UMR CNRS 6629-Université de Nantes, 2 Rue de la Houssinière, BP 92 208, F-44 322 Nantes Cedex 3, France

E-mail address: Luc.Hillairet@math.univ-nantes.fr 